\title{
Analysis of the role of microsomal triglyceride transfer protein in the liver of tissue-specific knockout mice
}

\author{
Martin Raabe, ${ }^{1,2}$ Murielle M. Véniant, ${ }^{1,2}$ Meghan A. Sullivan, ${ }^{1}$ Constance H. Zlot, ${ }^{1}$ \\ Johan Björkegren, ${ }^{1}$ Lars Bo Nielsen, ${ }^{1,2}$ Jinny S. Wong, ${ }^{2}$ Robert L. Hamilton,,${ }^{1,2,3}$ \\ and Stephen G. Young 1,2,4 \\ ${ }^{1}$ Gladstone Institute of Cardiovascular Disease, \\ ${ }^{2}$ Cardiovascular Research Institute, \\ ${ }^{3}$ Department of Anatomy, and \\ ${ }^{4}$ Department of Medicine, University of California-San Francisco, San Francisco, California 94143, USA \\ Address correspondence to: Stephen G. Young, Gladstone Institute of Cardiovascular Disease, PO Box 419100, San Francisco, \\ California 94141-9100, USA. Phone: (415) 826-7500; Fax: (415) 285-5632; E-mail: syoung@gladstone.ucsf.edu.
}

Martin Raabe’s present address is: Bayer AG, Business Group Pharma, Cardiovascular Research Institute, 42096 Wuppertal, Germany.

Lars Bo Nielsen's present address is: Department of Clinical Biochemistry, Rigshospitalet, University of Copenhagen, 2100

Copenhagen, Denmark.

Received for publication February 17, 1999, and accepted in revised form March 25, 1999.

\begin{abstract}
A deficiency in microsomal triglyceride transfer protein (MTP) causes the human lipoprotein deficiency syndrome abetalipoproteinemia. However, the role of MTP in the assembly and secretion of VLDL in the liver is not precisely understood. It is not clear, for instance, whether MTP is required to move the bulk of triglycerides into the lumen of the endoplasmic reticulum (ER) during the assembly of VLDL particles. To define MTP's role in hepatic lipoprotein assembly, we recently knocked out the mouse MTP gene (Mttp). Unfortunately, achieving our objective was thwarted by a lethal embryonic phenotype. In this study, we produced mice harboring a "floxed" Mttp allele and then used Cre-mediated recombination to generate liver-specific Mttp knockout mice. Inactivating the Mttp gene in the liver caused a striking reduction in VLDL triglycerides and large reductions in both VLDL/LDL and HDL cholesterol levels. The Mttp inactivation lowered apo B-100 levels in the plasma by $>95 \%$ but reduced plasma apo B-48 levels by only $\sim 20 \%$. Histologic studies in liver-specific knockout mice revealed moderate hepatic steatosis. Ultrastructural studies of wild-type mouse livers revealed numerous VLDL-sized lipid-staining particles within membrane-bound compartments of the secretory pathway (ER and Golgi apparatus) and few cytosolic lipid droplets. In contrast, VLDL-sized lipid-staining particles were not observed in MTPdeficient hepatocytes, either in the ER or in the Golgi apparatus, and there were numerous cytosolic fat droplets. We conclude that MTP is essential for transferring the bulk of triglycerides into the lumen of the ER for VLDL assembly and is required for the secretion of apo B-100 from the liver.
\end{abstract}

J. Clin. Invest. 103:1287-1298 (1999).

\section{Introduction}

Microsomal triglyceride transfer protein (MTP) is required for the secretion of apo B-containing lipoproteins from hepatocytes and from the absorptive enterocytes of the intestine $(1,2)$. MTP is also found in other tissues where apo $\mathrm{B}$-containing lipoproteins are synthesized, such as the myocardium (3, 4), yolk sac (5), and kidney (6). MTP is located within the lumen of the endoplasmic reticulum (ER), where it is assumed to transfer lipids during the assembly of lipoproteins (7). In in vitro systems, MTP transfers both neutral and polar lipids between liposomal membranes, but lipid transfer is most efficient with neutral lipids, (triglycerides and cholesterol esters). The MTP holoprotein is a heterodimer consisting of a $55-\mathrm{kDa}$ multifunctional protein (protein disulfide isomerase) and a unique $97-\mathrm{kDa}$ subunit. The $97-\mathrm{kDa}$ subunit confers all of the lipid transfer activity to the heterodimer $(8,9)$.

The importance of MTP for the secretion of apo B-containing lipoproteins was revealed in 1992, when Wetterau et al. (10) reported that both MTP activity and protein were absent in duodenal tissue of humans with abetalipoproteinemia. Abetalipoproteinemia is a recessive human disease characterized by intestinal fat malabsorption, deficiencies of fat-soluble vitamins, and a near-complete absence of apo B-containing lipoproteins (e.g., chylomicrons, VLDL, and LDL) in the plasma (11). Abetalipoproteinemia is caused by a variety of mutations in the gene for the 97-kDa subunit of MTP $(10,12-14)$.

Although human genetic studies have established the importance of MTP for the secretion of apo B-containing lipoproteins, and although in vitro studies have established that MTP can transfer lipids between liposomal membranes, the role of MTP in lipoprotein assembly and secretion has not yet been fully defined (15). The addition of lipids to apo $\mathrm{B}$ during the assembly of lipoproteins is widely believed to involve 2 steps (16-24). In the first step, small amounts of lipids are thought to be added to apo B as it is translated and translocated into the lumen of the rough ER, allowing apo B to fold correctly and avoid degradation. MTP is widely believed 

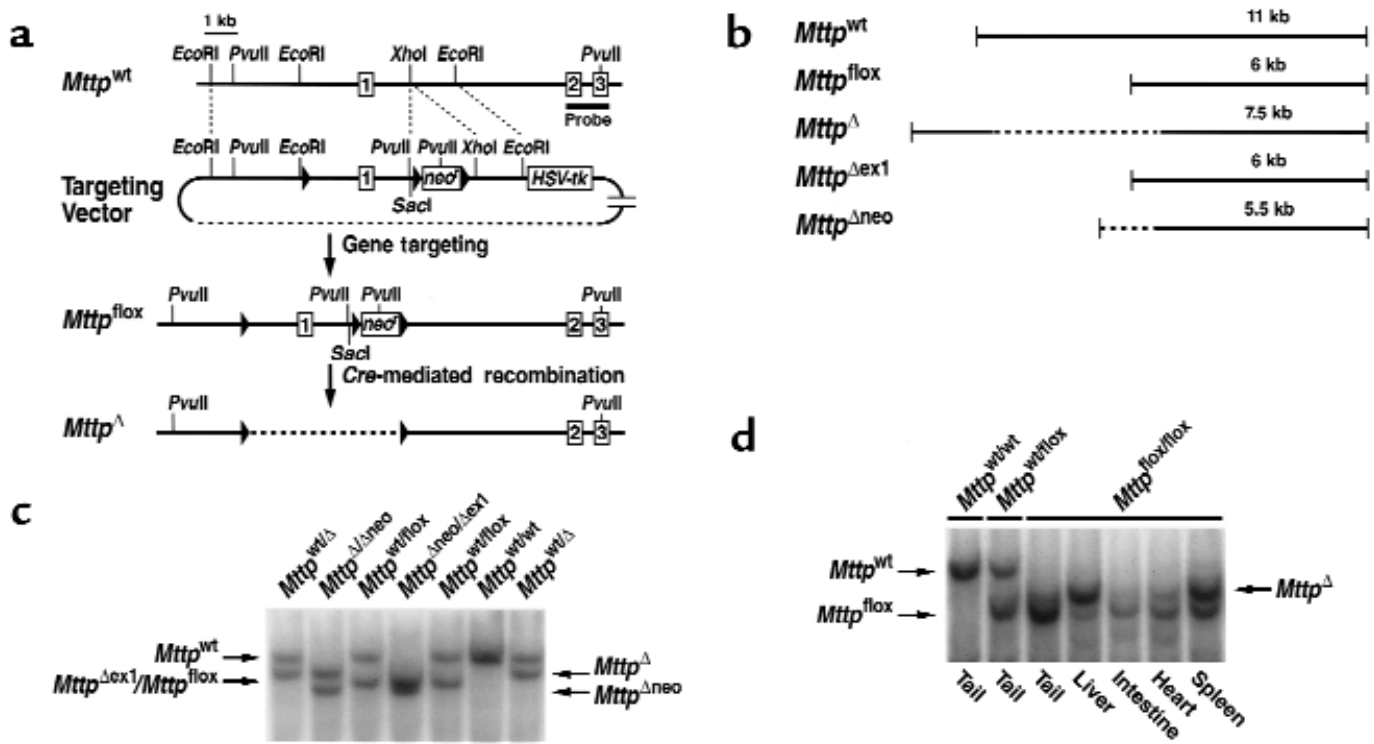

\section{Figure 1}

Generation and characterization of a floxed Mttp allele. (a) Schematic of the sequence-replacement gene-targeting strategy. The map of the wild-type Mttp allele (Mttp ${ }^{\text {wt }}$ ) spans the Mttp promoter (the sequences upstream from exon 1) and exons 1-3. The location of the 1.3-kb 3' flanking probe (5) is indicated. Recombination of the gene-targeting vector with the cognate sequences in the chromosomal DNA produces a mutant Mttp allele, Mttp flox, in which the promoter and exon 1 of the Mttp allele are flanked by loxP sites (filled triangles). An additional loxP site is located downstream from the neo. Cre-mediated excision of both the neo and the promoter/exon 1 fragment produces a null Mttp allele (designated Mttp ${ }^{\Delta}$ ). (b) Illustration of the Pvull fragments produced by different Cre-mediated recombination events: excision of both the neo and the promoter/exon 1 fragment (in an Mttp ${ }^{\Delta}$ allele), excision of the promoter/exon 1 fragment alone (in an Mttp ${ }^{\Delta x \times 1}$ allele), and excision of the neo alone (in an Mttp ${ }^{\Delta \text { neo }}$ allele). (c) A Southern blot of Pvull-digested genomic DNA from offspring of Mttpwt/flox/deleter-Cre intercrosses. The blot was hybridized with the $3^{\prime}$ flanking probe. Each of the 3 possible Cre-mediated recombination events was observed $\left(M t t p^{\Delta e x 1}, M t t p^{\Delta \text { neo }}\right.$, and $\left.M t t p^{\Delta}\right)$. Southern blots of Pvull-cleaved genomic DNA did not distinguish between the Mttp flox and Mttp ${ }^{\Delta \mathrm{ex} 1}$ alleles. The mouse Mttp gene contains Sacl sites located $2 \mathrm{~kb} 5^{\prime}$ and $2.5 \mathrm{~kb} 3^{\prime}$ to the gene fragment illustrated in a. The gene-targeting event introduced a new Sacl site. The recombination events could also be analyzed, therefore, with Sacl-cleaved genom-

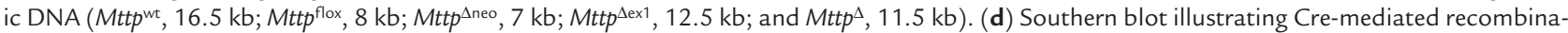
tion in different tissues of an Mttpflox/flox/Mx1-Cre mouse in which Cre expression had been induced with plpC. DNA samples from various tissues were digested with Pvull; the blot was hybridized with the 3' flanking probe.

to play an important role in this first "apo B lipidation" step $(1,21,25)$. The existence of apo B in the rough ER of hepatocytes has been documented by immunoelectron microscopy (16). However, the lipidated apo B particles cannot be seen in the rough ER by routine electron microscopy, even when the thin sections are stained for lipids, because those particles are too small $(<150 \AA$ in diameter) and lipid poor to be resolved by this technique. In a second step, the lipidated apo B molecule is thought to acquire the bulk of its core lipids by fusing with a large, VLDL-sized, apo B-free triglyceride particle (a "second-step" particle) $(17,23)$. The existence of the secondstep triglyceride particles within a special compartment of the smooth ER has been supported by 2 different electron microscopic studies $(16,23)$. Biochemical studies of VLDL assembly have also supported the concept that the bulk of neutral lipids are added to apo B in a second step after its translation is complete $(1,24)$.

The role, if any, of MTP in the formation of second-step lipid particles is unclear. In fact, this issue has recently been highlighted as one of the fundamental problems in understanding MTP and lipoprotein assembly $(15,26)$. In recent years, several groups have tried to address this issue by performing metabolic labeling/ pulse-chase studies of lipoprotein secretion from cultured McA-RH7777 cells (a rat hepatoma cell line) in the presence of MTP inhibitor drugs, but their conclusions were very different -indeed, almost opposite. Using an MTP inhibitor drug and complex metabolic labeling protocols, one group concluded that MTP had little or no role in the addition of the bulk of lipids to nascent apo B-48-containing lipoproteins (21). Using a similar approach and the same MTP inhibitor, another group reached the opposite conclusion - that MTP is essential for adding most of the triglycerides to apo B-containing VLDL during the second step of lipoprotein assembly (27).

We reasoned that MTP's role, if any, in the formation of second-step lipid particles within the lumen of the smooth ER might be resolved by ultrastructural studies of lipoprotein-producing tissues that were genetically deficient in MTP. If MTP's only role in lipoprotein assembly were to lipidate the apo B molecule as it was translocated into the lumen of the rough ER (i.e., the first lipidation step), one would expect that ultrastructural studies of MTP-deficient livers would reveal normal numbers of VLDL-sized, lipid-staining, second-step particles within the lumen of the smooth ER. On the other hand, if MTP were essential for the formation of the secondstep particles, one would expect to observe a virtual absence of lipoprotein-sized particles within all membrane-bound compartments of the secretory pathway.

Initially, we thought that it would be possible to investigate MTP's role in transferring the bulk of the core lipids into the lumen of the smooth ER by generating 
a

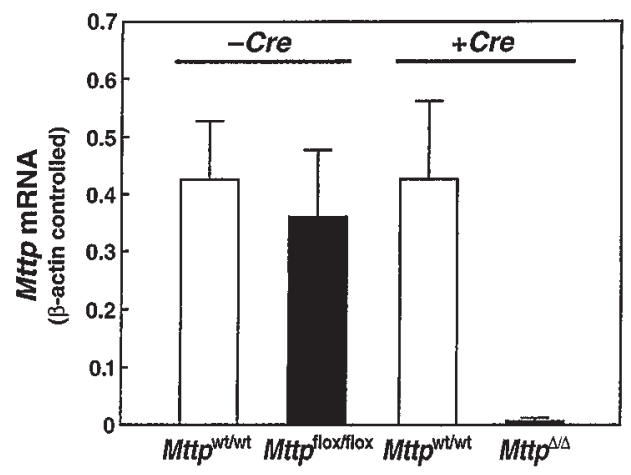

b

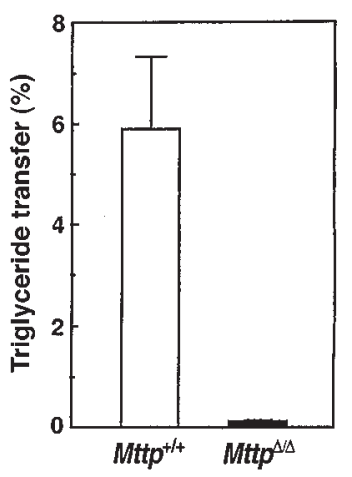

Figure 2

Inactivation of the Mttp gene in the liver. (a) Bar graphs illustrating Mttp mRNA levels in groups of Mttpt wt $^{\mathrm{wt}}$ and $M t t p^{\text {flox } / \text { flox }}$ mice (no Cre expression) ( $n=5$ in each group) and groups of Mttpwt/wt/Mx1-Cre and Mttp flox/flox/Mx1-Cre mice ( $n=5$ in each group) where Cre expression had been induced with plpC. Mttp mRNA levels were determined with $\beta$-actin-controlled RPAs. (b) A bar graph illustrating MTP activity levels in livers from groups $(n=5)$ of Mttp ${ }^{\mathrm{w} / \mathrm{wt}}$ and $M t t p^{\text {flox }} /$ flox mice that had been treated with Cre adenovirus. Each bar graph shows mean \pm SD.

MTP knockout mice $\left(\mathrm{Mttp}^{-/-}\right)$and then examining the livers of those mice by electron microscopy. Unfortunately, at least from the perspective of understanding hepatic lipoprotein assembly, the $\mathrm{Mttp}^{-/-}$mice died early during embryonic development (5). To move past that roadblock, in this study we have used a conditional gene inactivation system based on Cre/loxP technology $(28,29)$ to generate liver-specific Mttp knockout mice. A detailed characterization of those mice, including ultrastructural studies of lipoprotein assembly, has provided fresh insights into the role of MTP in lipoprotein assembly.

\section{Methods}

Generation of Mtt flox mice. A sequence-replacement gene-targeting vector was constructed from subclones of a $15-\mathrm{kb}$ SacI fragment spanning exons 1-3 of the mouse Mttp gene (ref. 5 and Figure 1a). A neomycin-resistance cassette (neo) flanked by loxP sites ("floxed") was inserted into intron 1 of the Mttp gene. An additional loxP site was inserted upstream from the Mttp promoter and exon 1 (2.5 kb 5' of exon 1) (Figure 1a). All 3 loxP sites were in the same orientation. The vector was electroporated into strain 129/SvJae embryonic stem cells, and drug-resistant clones were screened by Southern blot analysis. Several targeted clones were used to generate mice harboring a mutant $M t t p$ allele, $M t t p^{\text {flox }}$, in which exon 1 was flanked by loxP sites. All of the mice had a mixed genetic background ( $\sim 50 \%$ C57BL/6 and $\sim 50 \% 129 /$ SvJae). The mice were weaned at 21 days of age, housed in a barrier facility with a 12-hour light/12-hour dark cycle, and fed a chow diet containing $4.5 \%$ fat (Ralston Purina, St. Louis, Missouri, USA).

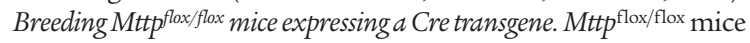
were bred with "deleter" Cre transgenic mice (30), in which Cre is expressed in the germ cells, and Mx1-Cre transgenic mice $(28,31)$. In Mx1-Cre transgenic mice, Cre expression can be induced in several tissues, including the liver, with a series of 3 intraperitoneal injections of $250 \mu \mathrm{g}$ of polyinosinic-polycytidylic ribonucleic acid (pIpC) (Sigma Chemical Co., St. Louis, Missouri, USA) (28).

Inactivating the Mttp gene in the liver with Cre adenovirus. The Mttp gene was inactivated in hepatocytes of $M t t p^{\text {flox/flox }}$ mice with an intravenous injection of $2 \times 10^{9}$ plaque-forming units of Cre adenovirus (32), as described by Rohlmann et al. (33). The Cre adenovirus was prepared in 293 cells as previously described (34).

$R$ Nase protection assays. To measure Mttp mRNA levels, we used an RNase protection assay (RPA) (RPA II; Ambion Inc., Austin, Texas, USA) with a 395-bp Mttp riboprobe (5). $\beta$-actin mRNA measurements were assessed with an 82-bp riboprobe at the same time, making it possible to normalize the $M t t p$ level to the $\beta$-actin signal. Apob mRNA levels were measured by RPAs with a 245-bp Apob riboprobe (35). Those levels were also normalized to $\beta$-actin.

Detection of apo B by Western blotting. Mouse plasma samples $(1.0 \mu \mathrm{L})$ were size-fractionated on a $4 \%$ polyacrylamide/SDS gel, and Western blots were performed with a rabbit antiserum against mouse apo B (35) and chemiluminescence detection reagents. The intensity of apo B-48 and apo B-100 bands on the $x$-ray films was determined by densitometry.

Measurements of lipids and apo B-100. Cholesterol and triglyceride levels in plasma samples were measured with enzymatic assays $(36,37)$. Phospholipids were measured with the phospholipid B assay (Wako Chemicals, Neuss, Germany) according to the manufacturer's protocol. The distribution of lipids in the plasma lipoprotein fractions was determined by fast-performance liquid chromatography (FPLC) (37). The concentration of mouse apo B-100 in mouse plasma was measured with an mAb-based sandwich RIA (38).

Table 1

Plasma lipid and apo B-100 levels

\begin{tabular}{|c|c|c|c|c|c|c|}
\hline \multirow{2}{*}{$\begin{array}{l}\text { Mttp genotype } \\
\text { Cre expression }\end{array}$} & \multicolumn{3}{|c|}{$M t t p^{w t / w t}$} & \multicolumn{3}{|c|}{$M t t p^{f l o x / f l o x}$} \\
\hline & None & Mx1-Cre + plpC & Cre adenovirus & None & Mx1-Cre + plpC & Cre adenovirus \\
\hline Number of mice & 6 & 6 & 5 & 6 & 6 & 5 \\
\hline Cholesterol (mg/dL) & $84 \pm 9$ & $87 \pm 6$ & $79 \pm 11$ & $70 \pm 9$ & $42 \pm 3^{A}$ & $38 \pm 6^{A}$ \\
\hline Triglycerides (mg/dL) & $79 \pm 12$ & $80 \pm 8$ & $76 \pm 10$ & $79 \pm 11$ & $30 \pm 6^{A}$ & $22 \pm 2^{A}$ \\
\hline Phospholipids (mg/dL) & $171 \pm 27$ & $158 \pm 50$ & ND & $140 \pm 24$ & $68 \pm 38^{A}$ & ND \\
\hline Apo B-100 (mg/dL) & $5.0 \pm 1.1$ & $5.7 \pm 0.1$ & $3.6 \pm 1.0$ & $4.4 \pm 0.9$ & $0.1 \pm 0.1^{\mathrm{A}}$ & $0.3 \pm 0.1^{\mathrm{A}}$ \\
\hline
\end{tabular}

Data represent means \pm SD. Blood was drawn from fasted female mice either 2 months after plpC treatment or 10 days after an intravenous injection of Cre adenovirus. AP $<0.001$ vs. Mttpwt/wt/Mx1-Cre mice by two-tailed unpaired $t$ test. ND, not determined. 
a

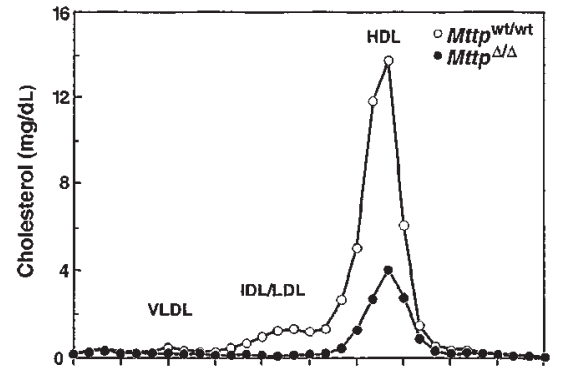

b

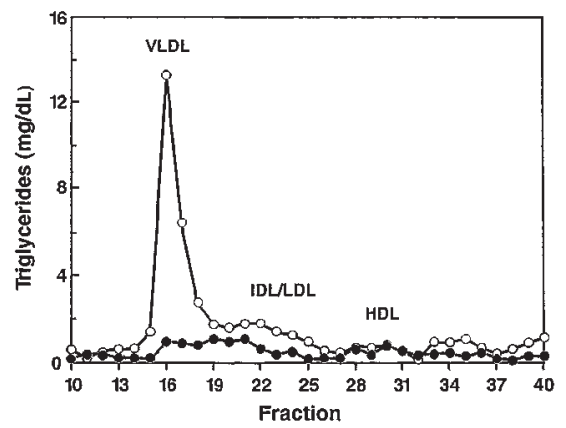

Figure 3

Distribution of cholesterol (a) and triglycerides (b) within the plasma lipoproteins of Mttpt ${ }^{\mathrm{wt} / \mathrm{wt}}$ and $\mathrm{Mtt} \mathrm{p}^{\Delta / \Delta}$ mice. Plasma $(200 \mu \mathrm{L})$ was pooled from 5 females of each genotype after a 4-hour fast and fractionated on an FPLC column $(36,37)$. The $M t t p^{\Delta / \Delta}$ mice were produced by treating Mttpflox/flox mice with Cre adenovirus. In the 2 experiments, the sum of the cholesterol levels in the HDL fractions of $M t t p^{\Delta \Delta}$ mice were reduced by $43 \%$ and $50 \%$ compared with those in $M t t p^{w t / w t}$ mice.

Metabolic labeling of mouse primary hepatocytes. Primary mouse hepatocytes were prepared as described previously (39). Hepatocytes $\left(6 \times 10^{5}\right)$ were plated onto collagen-coated 6-well tissue culture plates containing a mouse primary hepatocyte growth medium. The cells were allowed to attach for 1 hour, and the medium was removed and replaced with fresh medium containing $50 \mu \mathrm{L}$ of $\left[{ }^{35} \mathrm{~S}\right]$ methionine/cysteine (Pro-Mix; 530 $\mathrm{MBq} / \mathrm{mL}, 14.3 \mathrm{mCi} / \mathrm{mL}$; Amersham Life Sciences, Arlington Heights, Illinois, USA). After a 3-hour incubation, the apo B in the medium was immunoprecipitated with a mouse apo B-specific antiserum (35). The immunoprecipitates were sizefractionated on a $3-15 \%$ SDS-polyacrylamide gel. The gel was dried, and the relative amounts of ${ }^{35} \mathrm{~S}$ incorporation into apo B-48 and apo B-100 were assessed by autoradiography. The MTP activity in primary hepatocytes was measured as described previously $(7,10)$.
Histologic and ultrastructural analysis of tissues. Paraffin-embedded sections were stained with hematoxylin and eosin and periodic acid-Schiff (PAS). Frozen sections of intestinal and liver tissue were stained with oil red $\mathrm{O}$ and examined by light microscopy. For osmium tetroxide staining of lipids, tissue samples were embedded in epoxy resin after perfusion fixation with $1.5 \%$ glutaraldehyde, $4 \%$ polyvinylpyrrolidone (molecular weight 10,000 ), and $0.05 \%$ calcium chloride in $0.1 \mathrm{M}$ sodium cacodylate buffer ( $\mathrm{pH}$ 7.4). The osmium tetroxide-stained sections were counterstained with toluidine blue. Electron microscopy was performed on perfusion-fixed tissues that had been stained with the imidazole-buffered osmium tetroxide procedure $(23,40,41)$. In situ hybridization was performed as described (42) with a ${ }^{35}$ S-labeled Mttp riboprobe (5).

\section{Results}

Generating and characterizing mice with a floxed Mttp allele. Three loxP sites, two flanking a neo in intron 1 and a third $2.5 \mathrm{~kb}$ upstream from exon 1 , were introduced into the Mttp gene with a sequence-replacement gene-targeting vector (Figure 1a). Two targeted clones that retained all 3 loxP sites were identified, and one was used to produce chimeric mice. The chimeras were bred to generate mice that were heterozygous for the floxed Mttp allele (Mttp flox). The heterozygous mice (Mttpwt/flox) were intercrossed to produce homozygotes (Mttpflox/flox). The Mtt $p^{\text {flox/flox }}$ mice developed normally and were healthy and fertile.

Cre recombinase deletes the DNA sequences between loxP sites when the loxP sites are in the same orientation (43). Because there were 3 loxP sites in the Mttpflox allele, there were 3 possible Cre-mediated recombination events: deletion of the neo alone (generating an Mttp ${ }^{\Delta \text { neo }}$ allele), deletion of the promoter and exon 1 of the Mttp gene but not the neo (generating an $M t t t^{\Delta \mathrm{ex} 1}$ allele), and deletion of both the promoter/exon 1 segment and the neo (generating an $M t t p^{\Delta}$ allele) (Figure 1b). To establish that each of these recombination events could occur, we bred the Mttplox/flox mice with deleter-Cre transgenic mice (29). An analysis of offspring from $M t t p^{f l o x / w t} /$ deleter-Cre intercrosses revealed germline separation of 5 different Mttp alleles (Mttp ${ }^{w t}$, Mttp flox, $M t t p^{\Delta \text { neo }}, M t t p^{\Delta e x 1}$, and $M t t p^{\Delta}$ ) (Figure 1c). All of the weaned offspring had 1 functional $M t t p$ allele (i.e., $M t t p^{\mathrm{wt}}$, $M t t p^{f l o x}$, or $\left.M t t p^{\Delta \text { neo }}\right)$ - an expected finding, given that homozygosity for an Mttp null allele causes lethal developmental abnormalities (5).

Table 2

Serum chemistries

\begin{tabular}{|c|c|c|c|c|c|}
\hline Mttp genotype & $M t t p^{w t / w t}$ & $M t t p^{w t / w t}$ & Mttp flox/flox & $M t t p^{w t / w t}$ & $M t t p^{f l o x / f l o x}$ \\
\hline Cre expression & none & Mx1-Cre + plpC & Mx1-Cre + plpC & Cre adenovirus & Cre adenovirus \\
\hline Number of mice & 8 & 5 & 3 & 1 & 1 \\
\hline $\operatorname{ALT/SGPT~(IU/L)~}$ & $26 \pm 6$ & $25 \pm 6$ & $19 \pm 1$ & ND & ND \\
\hline AST/SGOT (IU/L) & $86 \pm 15$ & $48 \pm 1$ & $52 \pm 6$ & 1,044 & 885 \\
\hline Albumin (g/dL) & $2.2 \pm 0.1$ & $2.3 \pm 0.1$ & $2.5 \pm 0.1$ & 3 & 2.4 \\
\hline Total protein $(\mathrm{g} / \mathrm{dL})$ & $3.8 \pm 0.1$ & $4.3 \pm 0.1$ & $4.2 \pm 0.2$ & 6.6 & 4.8 \\
\hline Bilirubin (mg/dL) & 0 & $0.2 \pm 0.1$ & $0.2 \pm 0.1$ & 0 & 0 \\
\hline $\mathrm{BUN}(\mathrm{mg} / \mathrm{dL})$ & $14 \pm 1$ & $19 \pm 1$ & $20 \pm 3$ & 21 & 36 \\
\hline Creatinine (mg/dL) & $0.2 \pm 0.1$ & $0.3 \pm 0.1$ & $0.3 \pm 0.1$ & ND & ND \\
\hline Glucose (mg/dL) & $175 \pm 8$ & $204 \pm 17$ & $188 \pm 13$ & ND & ND \\
\hline
\end{tabular}

Data represent means \pm SD. Blood was drawn either 2 months after plpC treatment or 10 days after the Cre adenovirus injection. ND, not determined. 
a

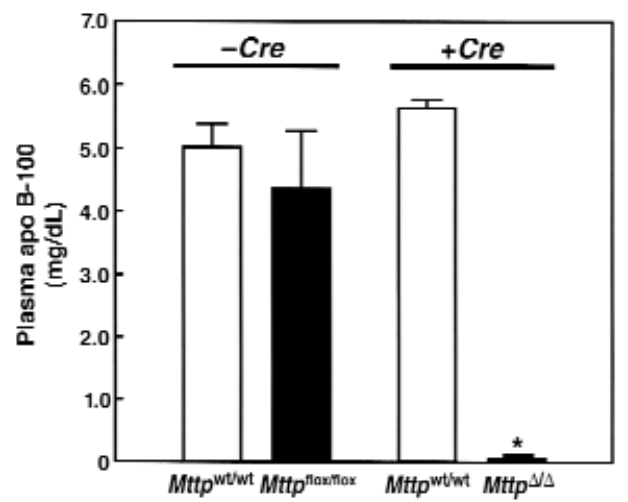

b

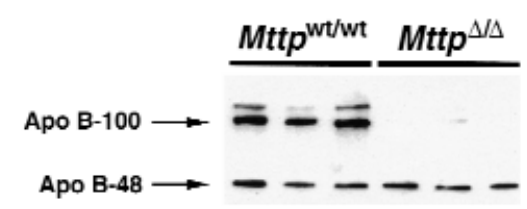

\section{Figure 4}

Plasma apo B levels in Mttpwt/wt and Mttp $p^{\Delta / \Delta}$ mice. (a) Bar graphs illustrating apo B-100 concentrations (means \pm SD) in the plasma of $M t t p^{w / / w t} / \mathrm{Mx} 1-\mathrm{Cre}$ and Mttp flox/flox/Mx1-Cre mice, both before and after plpC induction of Cre expression. Apo B-100 concentrations were assessed with an mAb-based RIA. (b) Analysis of plasma apo B levels in $M t t p^{\Delta \Delta}$ mice (Mttf flox/flox/Mx1-Cre mice treated with $\left.\mathrm{plpC}\right)$, as judged by Western blots of SDS-polyacrylamide gels. Plasma samples (obtained after a 4-hour fast) were size-fractionated on a $4 \%$ SDS-polyacrylamide gel, and Western blots were performed with a rabbit antiserum specific for mouse apo B (35).

Cre-mediated recombination was also evident after the induction of Cre expression from the Mx1-Cre transgene (28). Southern blots of pIpC-treated $M t t p^{\text {flox/flox } / M x 1-C r e ~}$ mice revealed that most of the $M t t p^{\text {flox }}$ alleles in the liver were converted to $M t t p^{\Delta}$ alleles. High levels of recombination were also noted in the spleen; lesser degrees of recombination were observed in the small intestine and heart (Figure 1d).

Inactivating the Mttp gene in the liver. To generate mice lacking Mttp gene expression in the liver, we used 2 different approaches. First, we injected Cre adenovirus into an internal jugular vein of $M t t p^{\text {flox/flox }}$ mice. Second, we bred the Mx1-Cre transgene onto the background of homozygosity for the Mtt $p^{\text {flox }}$ allele and then induced Cre expression with pIpC.

The Mttp mRNA levels were essentially identical with $M t t p^{\text {flox }}$ and $M t t p^{\mathrm{wt}}$ alleles; in the absence of Cre, there were no statistically significant differences in Mttp mRNA levels in $M t t p^{\text {flox/flox }}$ and $M t t p^{\mathrm{wt} / \mathrm{wt}}$ mice, either in the intestine (not shown) or in the liver (Figure 2a). However, when Cre expression in Mttplox/flox /Mx1-Cre transgene mice was induced with $\mathrm{pIpC}$ (to generate $M t t p^{\Delta / \Delta}$ mice), $M t t p$ mRNA levels fell by $96 \pm 3 \%$ compared with those in PIpC-treated $M t t p^{\mathrm{wt} / \mathrm{wt}} / \mathrm{Mx} 1-\mathrm{Cre}$ mice $(P=0.002)$ (Figure 2a). Similarly, Mttp mRNA levels were $98 \pm 3 \%$ lower in Cre adenovirus-treated $M t t p^{\text {flox/flox }}$ mice than in Cre adenovirus-treated $M t t p^{\mathrm{wt} / \mathrm{wt}}$ mice $(P=0.008)$. To test whether the Cre-induced decrease in Mttp mRNA levels resulted in a comparable reduction in MTP activity, we measured MTP activity levels in hepatocyte extracts.
Hepatic MTP activity was $97 \pm 5 \%$ lower in $M t t p^{\Delta / \Delta}$ mice than in the control group (Figure 2b). Based on the Mttp mRNA levels and MTP activity assays, as well as the Southern blots (Figure 1d), we concluded that both the Mx1-Cre transgene and Cre adenovirus approaches inactivated the Mttp gene in the majority (>95\%) of hepatocytes.

Decreased plasma lipid levels in mice lacking hepatic Mttp gene expression. As expected, the plasma lipid levels in $M t t p^{\text {flox/flox }}$ and $M t t p^{\mathrm{wt} / \mathrm{wt}}$ mice were similar (Table 1). However, after $\mathrm{pIpC}$ induction of Cre expression in $M t t p^{\text {flox/flox} / M x 1-C r e ~ m i c e, ~ t h e ~ t o t a l ~ p l a s m a ~ c h o l e s t e r o l ~}$ levels were only $48 \pm 5 \%$ of those in pIpC-treated

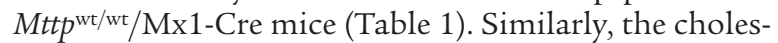
terol levels in adenovirus-treated $M t t \mathrm{p}^{\text {flox/flox }}$ mice were only $48 \pm 8 \%$ of those observed in adenovirus-treated $M t t p^{\mathrm{wt} / \mathrm{wt}}$ mice. In pIpC-treated Mttp flox/flox/Mx1-Cre transgenic mice and $M t t p^{\text {flox/flox }}$ mice treated with Cre adenovirus, the plasma triglycerides fell by $62 \%$ and $72 \%$, respectively. The absence of Mttp expression in the liver profoundly affected the distribution of lipids within the plasma lipoproteins (Figure 3). In $M t t p^{\Delta / \Delta}$ mice, cholesterol was virtually undetectable in the VLDL and LDL fractions and was reduced by $\sim 50 \%$ in the HDL fraction (Figure 3a). The amount of triglycerides in the VLDL fraction was strikingly reduced in the $M t t p^{\Delta / \Delta}$ mice (Figure 3b).

The effects of hepatic Mttp gene expression on plasma apo $B$ levels. As judged by a mAb-based RIA, apo B-100 levels fell by $99 \pm 1 \%$ in the $M t t p^{\text {flox/flox }} / \mathrm{Mx} 1$-Cre mice after $\mathrm{pIpC}$ induction but did not change in pIpC-treated $M t t p^{w t / w t} / \mathrm{Mx} 1-C r e$ mice (Figure. 4a). Apo B-100 levels were reduced to a similar extent $(91 \pm 5 \%)$ in Cre adenovirus-treated $M t t p^{\text {flox/flox }}$ mice. The striking reduction in plasma apo B-100 levels was confirmed by Western blotting (Figure 4b).

Interestingly, the induction of hepatic MTP deficiency did not have a major effect on the plasma levels of apo B48 (Figure 4b). Scanning of Western blots with a densitometer revealed that apo B-48 levels in $M t t p^{\Delta / \Delta}$ mice $(n=$ 13) fell by $20 \%$, a difference that was not statistically significant $(P=0.23)$. The plasma apo B-48 levels were variable. Occasionally, we observed animals in which the plasma apo B-48 levels were reduced by $\sim 50 \%$, but we also observed many animals in which there was no change in the apo B-48 levels. Although the plasma levels of apo B-48 were reduced only moderately in $M t t p^{\Delta / \Delta}$ mice, there were striking reductions in the levels of apo B-48 in the VLDL. Using a densitometric analysis of the distribution of apo B-48 in the FPLC fractions, we found that $30 \%$ of the apo B-48 in $M t t p^{\mathrm{wt} / \mathrm{wt}}$ mice was in the VLDL fractions, while $70 \%$ was in the LDL and HDL fractions. In the $M t t p^{\Delta / \Delta}$ mice, $99.3 \%$ of the apo B-48 was in the LDL and HDL fractions and only $0.7 \%$ was in the VLDL fractions.

The fact that hepatic Mttp deficiency caused a striking reduction in the plasma levels of apo B-100, but not apo B-48, led us to consider the hypothesis that hepatic MTP deficiency might affect the secretion of apo B-100 and apo B-48 differently, i.e., that MTP might be critical for apo B-100 secretion, but less important for apo B-48 secretion. To address that hypothesis, we isolated primary hepatocytes from $M t t p^{\Delta / \Delta}$ and $M t t p^{w t / w t}$ mice and 
a

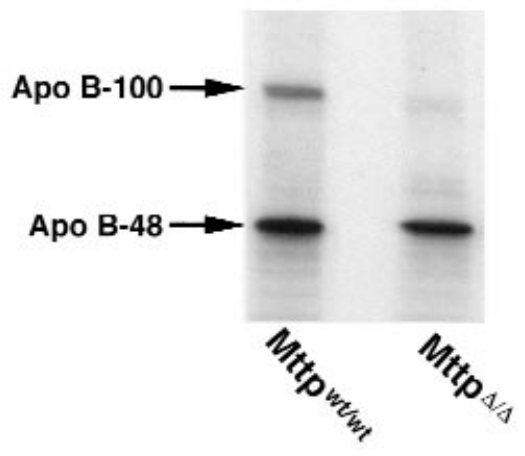

b

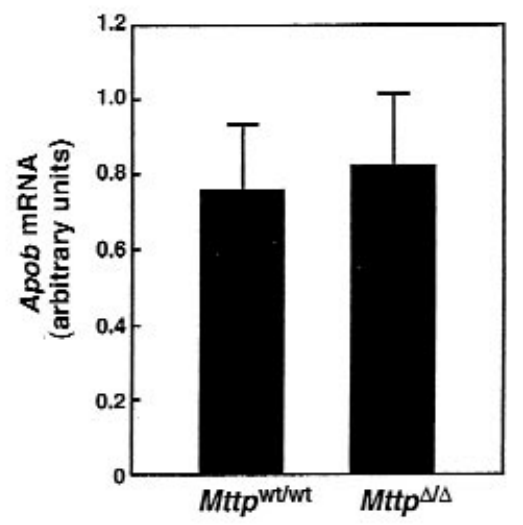

Figure 5

Apo B secretion and Apob mRNA levels in Mttp ${ }^{\Delta / \Delta}$ mice. (a) Apo B accumulation in the medium from $M t t p^{\text {wt/wt }}$ and $M t t p^{\Delta / \Delta}$ primary hepatocytes. The $M t t p^{\Delta \Delta}$ mice were produced by treating Mttp flox/flox mice with Cre adenovirus. Primary hepatocytes were grown in the presence of $\left[{ }^{35} \mathrm{~S}\right]$ methionine/cysteine; the apo B proteins were then immunoprecipitated and resolved on SDS-polyacrylamide gels. Large amounts of apo B-48 secretion from $M t t p^{\Delta \Delta}$ primary hepatocytes were observed in this experiment and in 2 subsequent experiments. (b) A bar graph illustrating Apob mRNA levels in the livers of $M t t p^{\mathrm{wt} / \mathrm{wt}}$ and $M t t p^{\Delta / \Delta}$ mice $(n=10$ in each group; $P=$ $0.54)$. The $M t t p^{\Delta \Delta}$ mice were produced by treating $M t t p^{\text {flox }} /$ flox $/ M \times 1-C r e$ mice with $\mathrm{plpC}$. The bar graph shows mean \pm SD.

incubated them in the presence of $\left[{ }^{35} \mathrm{~S}\right]$ methionine/cysteine. MTP activity was reduced by $98 \%$ in the $M t t p^{\Delta / \Delta}$ hepatocytes, compared with the Mttp $\mathrm{wt}^{\mathrm{wt}}$ hepatocytes (data not shown). The amount of apo B-100 in the medium of $M t t p^{\Delta \Delta}$ hepatocytes was substantially lower than the amount of apo B-100 from $M t t p^{\mathrm{wt} / \mathrm{wt}}$ hepatocytes (Figure 5a). In contrast, there was little change in the amount of apo B-48 in the medium.

Normal apo B-100 mRNA levels in the livers of mice lacking hepatic Mttp gene expression. Several groups have reported elevated apo B mRNA levels in the tissues of humans with abetalipoproteinemia (44-46), raising the possibility of feedback upregulation of apo B synthesis in the setting of diminished lipoprotein secretion. To test whether hepatic MTP deficiency truly results in an upregulation of apo B mRNA levels, we measured Apob mRNA levels in $M t t p^{\Delta / \Delta}$ and $M t t p^{\mathrm{wt} / \mathrm{wt}}$ mice with a $\beta$-actin-controlled RPA. The Apob mRNA levels in the 2 groups of mice were identical (Figure 5b).

Accumulation of cytosolic lipid droplets in mice lacking hepatic Mttp expression. The absence of MTP in the liver resulted in hepatic steatosis. The livers from $M t t p^{\mathrm{wt} / \mathrm{wt}}$ mice were beefy red; the livers from $M t t p^{\Delta / \Delta}$ mice were predominantly red, but had a slight yellowish hue. In osmium tetroxide-stained sections, the $M t t p^{\mathrm{wt} / \mathrm{wt}}$ hepatocytes were nearly free of intracellular lipid droplets; most of the lipid staining was confined to the vitamin A-storing Ito cells (Figure 6a). In contrast, the vast majority of hepatocytes from $M t t p^{\Delta / \Delta}$ mice contained multiple fat droplets (Figure 6b). Of note, an occasional hepatocyte (perhaps $\sim 2 \%$ of the total) appeared to be free of fat droplets; we suspect that those cells harbored an Mttp flox allele that had not been converted to an $M t t p^{\Delta}$ allele. The conclusion that the occasional "normal appearing" hepatocytes probably did not have an $M t t p^{\Delta \Delta}$ genotype is supported by the Mttp mRNA assays and MTP activity assays shown in Figure 2. Those assays revealed that we eliminated Mttp gene expression by $>95 \%$, but not by $100 \%$. Two other histologic findings from the livers of $M t t p^{\Delta \Delta \Delta}$ mice were noteworthy. First, staining with PAS revealed decreased amounts of glycogen in the livers of $M t t p^{\Delta / \Delta}$ mice (data not shown). Second, there was no evidence of inflammation (e.g., an infiltration of lymphocytes or neutrophils) in the livers of $M t t p^{\Delta \Delta}$ mice (pIpCtreated $M t t p^{\text {flox/flox} / M x 1-C r e ~ m i c e) ~(F i g u r e ~ 6 b) . ~}$ Consistent with that observation, liver function in $M t t p^{f l o x} /$ lox $/ \mathrm{Mx} 1$-Cre mice was normal 2 months after pIpC treatment (Table 2). In contrast, the serum transaminases were elevated 10 days after injection of the Cre adenovirus (Table 2).

Limited expression of the Mx1-Cre transgene in the duodenum. The Mttp gene is expressed at high levels within the villus enterocytes of the intestine (Figure 6c). Southern blots of intestinal DNA from pIpC-treated $M t t \mathrm{flox}^{\mathrm{fl} / \mathrm{flox}} / \mathrm{Mx} 1-\mathrm{Cre}$ mice indicated that $\sim 30 \%$ of the $M t t p^{\text {flox }}$ alleles had been converted to $M t t p^{\Delta}$ alleles (Fig. 1d). Since each intestinal villus is formed from only a few stem cells within the crypts $(47,48)$, we reasoned that the generation of $M t t p^{\Delta \Delta}$ stem cells might lead to patches of fatty enterocytes on some intestinal villi. This suspicion was confirmed. In osmium tetroxide-stained sections of the duodenum from $\mathrm{pIpC}$-treated $\mathrm{Mtt} \mathrm{p}^{\mathrm{flox} / \mathrm{flox}} / \mathrm{Mx} 1-\mathrm{Cre}$ mice, $\sim 20 \%$ of the villi had well-circumscribed patches of fatty enterocytes (Figure $6 \mathrm{~d}$ and e). These patches were observed only within the lower half of the intestinal villi and never in $M t t p^{\mathrm{wt} / \mathrm{wt}}$ mice (Figure 6f).

Ultrastructural analysis of mice lacking Mttp gene expression in the liver. To better define the role of MTP in the assembly of lipoproteins in the liver, we analyzed liver tissue from $\mathrm{pI}$ C-treated $M t t p^{\text {flox } / \text { flox }} / \mathrm{Mx} 1-\mathrm{Cre}$ and $M t t p^{\mathrm{wt} / \mathrm{wt}}$ mice by electron microscopy. Each $M t t p^{\mathrm{wt} / \mathrm{wt}}$ hepatocyte contained numerous VLDL-sized lipid-staining particles in the membrane-bound compartments of the secretory pathway (e.g., smooth ER and Golgi apparatus) (Figure 7). As previously described by Alexander et al. (16), we observed that some of the lipid-staining particles in the ER were located in a terminal outpouching of that organelle. Golgi stacks and secretory vesicles in $M t t \mathrm{p}^{\mathrm{w} / \mathrm{wt}}$ hepatocytes were filled with large, VLDL-sized lipoproteins, typically in grapelike clusters (Figure 7).

There was clear-cut heterogeneity in the ultrastructural appearance of hepatocytes from pIpC-treated Mttp plox/flox/Mx1-Cre mice, almost certainly because a small percentage of the cells $(\sim 2 \%)$ had not been con- 

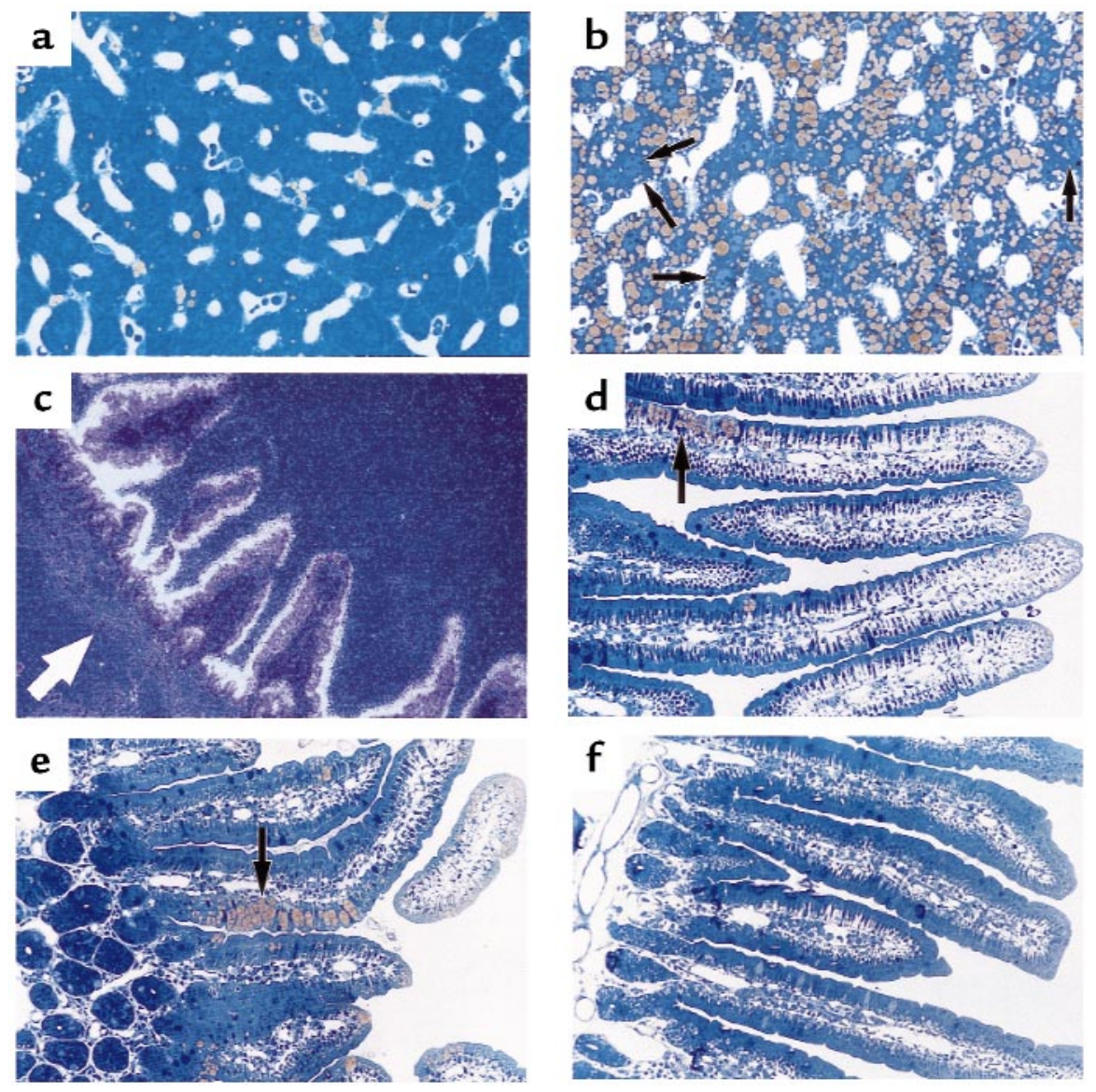

Figure 6

Liver and intestinal histology in Mttpwt/wt and Mttp ${ }^{\Delta \Delta}$ mice. (a) Osmium tetroxide-stained section of the liver of an Mttp ${ }^{w t / w t}$ mouse. (b) Osmium tetroxide-stained section of the liver of an $M t t p^{\Delta \Delta}$ mouse (produced by treating Mttp flox/flox/Mx1-Cre mice with plpC). The yellow staining represents intracellular fat droplets. Arrows point to rare hepatocytes that do not appear to have a significant amount of cytosolic fat droplets. (c) In situ hybridization of the duodenum from a wild-type mouse, demonstrating MTP expression in the villus enterocytes. The location of the crypt cells are denoted by a white arrow. (d and e) Osmium tetroxide-stained section of the duodenum from 2 different $M t t p^{\Delta / \Delta}$ mice). Discrete patches of lipid-filled villus enterocytes (arrows) were observed and were confined to the lower half of the villus. The MTP activity level in the intestine was $\sim 60 \%$ lower in plpC-

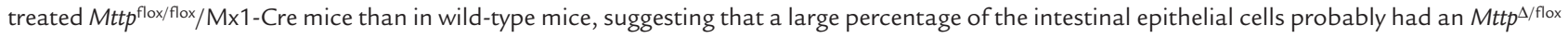
genotype. (f) Osmium tetroxide-stained section of the duodenum from an Mttpwt/wt mouse.

verted to the $M t t p^{\Delta \Delta}$ genotype. Relatively rare hepatocytes in these livers, perhaps 1 in $50-100$, had few or no cytosolic fat droplets and somewhat smaller VLDL-sized lipoproteins in the secretory pathway (Figure 8). In most $M t t p^{\Delta / \Delta}$ hepatocytes, however, there were many cytosolic lipid droplets and a complete absence of VLDL-sized lipid-staining particles in the ER and Golgi apparatus. In Figure 8, two adjacent hepatocytes illustrate this heterogeneity: the cell on the right side of the bile canaliculus (almost certainly an $M t t p^{\Delta \Delta}$ cell) had large, cytosolic lipid droplets and no round, VLDL-sized lipoproteins in the ER or Golgi apparatus. The cell on the left side of the bile canaliculus (likely an $M t t p^{\Delta / \text { flox }}$ or $M t t p^{\text {flox/flox }}$ cell) had few or no cytosolic lipid droplets and many lipoproteins in the Golgi apparatus. Figure 9 shows an electron micrograph of a typical $M t t p^{\Delta / \Delta}$ hepatocyte. Once again, there were no VLDL-sized, lipid-staining particles in any membrane-bound structure of the secretory pathway. Although VLDL-sized, round, lipid-staining particles were virtually never observed in the secretory organelles of $M t t p^{\Delta \Delta}$ cells, we occasionally observed small and irreg- ularly shaped lipid-staining particulate matter within the Golgi apparatus of those cells, the nature of which is presently unknown (see arrows in Figure 9).

\section{Discussion}

In this study, we used Cre/loxP recombination techniques to produce mice that lack Mttp expression in the liver. Certain aspects of the phenotype of these mice (e.g., their extremely low LDL cholesterol levels) were fairly predictable, given the well-documented plasma lipid abnormalities in humans with abetalipoproteinemia (11, 15). However, other features of MTP deficiency, particularly those related to MTP's role in lipoprotein assembly, were not as easy to predict. One of the most perplexing issues in MTP biology is whether MTP is essential for moving the bulk of neutral lipids into the lumen of the ER during the assembly of VLDL $(15,26)$. In this study, we provide compelling evidence that MTP plays a critical role in the movement of triglycerides into the lumen of the ER. In the absence of MTP, VLDL-sized, round, lipid-staining particles were absent in both the ER and 


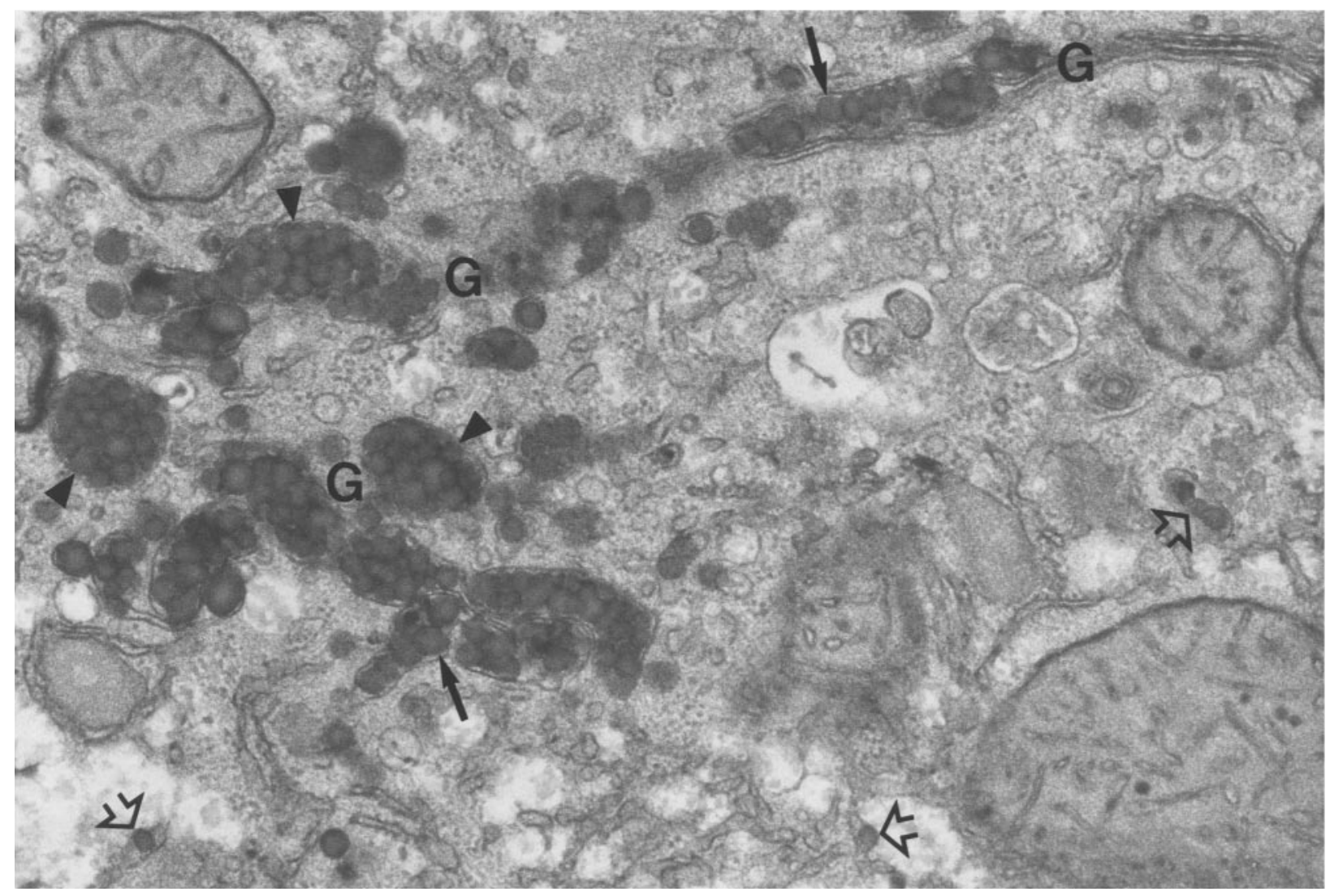

\section{Figure 7}

Electron micrograph of the Golgi apparatus region of an Mttpwt/wt hepatocyte, illustrating nascent VLDL particles in the smooth ER (open arrows), in the Golgi stacks (arrows), and in grapelike clusters within forming Golgi apparatus secretory vesicles (arrowheads). The majority of the particles in this image are 800-1,400 A. We examined more than 50 electron micrographs of Mttp ${ }^{\mathrm{wt} / \mathrm{wt}}$ hepatocytes; VLDL-sized particles were observed in the ER and Golgi of each and every hepatocyte. Some of the Mttp ${ }^{\text {wt/wt }}$ hepatocytes had smaller VLDL particles $(400-800 \AA)$ than illustrated in this figure. $\times 36,000$.

in the Golgi apparatus. The absence of triglycerides in the hepatocyte ER in the liver-specific Mttp knockout mice cannot be attributed to a paucity of lipids because the cytosol of those hepatocytes contained many large triglyceride droplets.

The absence of VLDL-sized lipid particles in the ER broadens our conception of MTP's role in lipoprotein assembly. In addition to "lipidating" apo B as it enters the lumenal space of the $\operatorname{ER}(15,26)$, MTP is critical for moving the bulk of neutral lipids into the lumen of the smooth ER for VLDL assembly. This broader view is in apparent conflict with a prior study by Gordon et al. (21). They used complex metabolic labeling protocols in cultured hepatoma cells to examine VLDL assembly and secretion, both in the absence and in the presence of MTP inhibitor drugs. They concluded that the addition of MTP inhibitor drugs to the cell-culture medium did not block the capacity of small and dense $(d>1.070$ $\mathrm{g} / \mathrm{mL}$ ) apo B-48 particles to acquire a bolus of lipids, thereby transforming them into large and buoyant $(d<$ $1.006 \mathrm{~g} / \mathrm{mL}$ ) VLDL. These metabolic labeling studies raised the possibility that MTP inhibitors might not block the formation of triglyceride-rich second-step particles within the ER. In view of our experiments that reveal that Mttp gene expression is required to move bulk amounts of triglycerides into the lumen of the smooth
ER, how can the prior metabolic labeling studies (21) be interpreted? We do not know the answer to this question with certainty, but we suspect that the conversion of apo B-48-containing HDL to apo B-48-containing VLDL in their experiments involved the acquisition of lipids that existed in the lumen of the ER before the addition of MTP inhibitor drugs.

In our experiments, both the Cre adenovirus approach and the Mx1-Cre transgene approach inactivated Mttp gene expression in $>95 \%$, but not $100 \%$, of hepatocytes. After the Cre expression, RNase protection assays revealed $>95 \%$ reductions in Mttp mRNA levels, but trace levels of the mRNA could still be detected. Consistent with that finding, light microscopy showed occasional hepatocytes with few cytosolic fat droplets. In those "nonfatty" cells, electron microscopy revealed robust VLDL assembly. Why were we unsuccessful in inactivating both copies of the Mttp gene in all hepatocytes? We suspect that the excision of a floxed segment of DNA is a stochastic event and that rare hepatocytes either do not express Cre (perhaps because of variegation in transgene expression) or simply escape the period of high-level Cre expression without both alleles' undergoing the gene-excision event. In a few experiments, we have administered Cre adenovirus to $M t t p^{\text {flox/flox} / M x 1-C r e ~ m i c e ~ i n ~ w h i c h ~ C r e ~ e x p r e s s i o n ~ h a d ~}$ 
already been induced with $\mathrm{pIpC}$ and found that the adenovirus further decreased Mttp gene expression (Raabe, M., et al., unpublished observations).

The plasma levels of apo B-100 and apo B-48 in the liver-specific Mttp knockout mice were intriguing. As judged by sensitive Western blots and mAb-based RIAs, the plasma levels of apo B-100 fell by $95 \%$ or more, a magnitude that was essentially identical to the decrease in Mttp mRNA levels and MTP activity levels and similar to the estimated percentage of cells containing many cytosolic fat droplets. In contrast, the plasma levels of apo B-48 were reduced by only $20 \%$. Our initial reaction to these results was to conclude that we had generated "intestinal lipoprotein only" mice and that all of the apo B-48 in the plasma originated in the intestine. That supposition was based on the fact that the intestine produces only apo B-48 (49) and the assumption that we had inactivated all apo B secretion by the liver. We then recalled that the plasma apo B-48 levels in mice lacking intestinal apo B synthesis are not appreciably lower than those in control mice that do synthesize intestinal lipoproteins (41). That observation, which indicated that most of the apo B-48 in mouse plasma originates from the liver, led us to consider an alternative explanation that a significant amount of apo B-48 might be secreted from hepatocytes in the absence of MTP. That hypothesis was strongly supported by our experiments with $M t t p^{\Delta / \Delta}$ primary hepatocytes, which showed a large amount of apo B-48 secretion in the setting of a $98 \%$ reduction in MTP activity levels.

High levels of apo B-48 secretion by $M t t p^{\Delta / \Delta}$ hepatocytes posed a conundrum because we had demonstrated by electron microscopy that the Golgi stacks were devoid of VLDL-sized lipoproteins. One way to reconcile the absence of VLDL in the Golgi stacks with abundant apo B-48 secretion from $M t t p^{\Delta / \Delta}$ primary hepatocytes is to hypothesize that Mttp-deficient hepatocytes secrete apo B48 on very small, dense, HDL-sized particles. While VLDL particles can be clearly viewed in the Golgi apparatus or ER in thin sections by transmission electron microscopy,

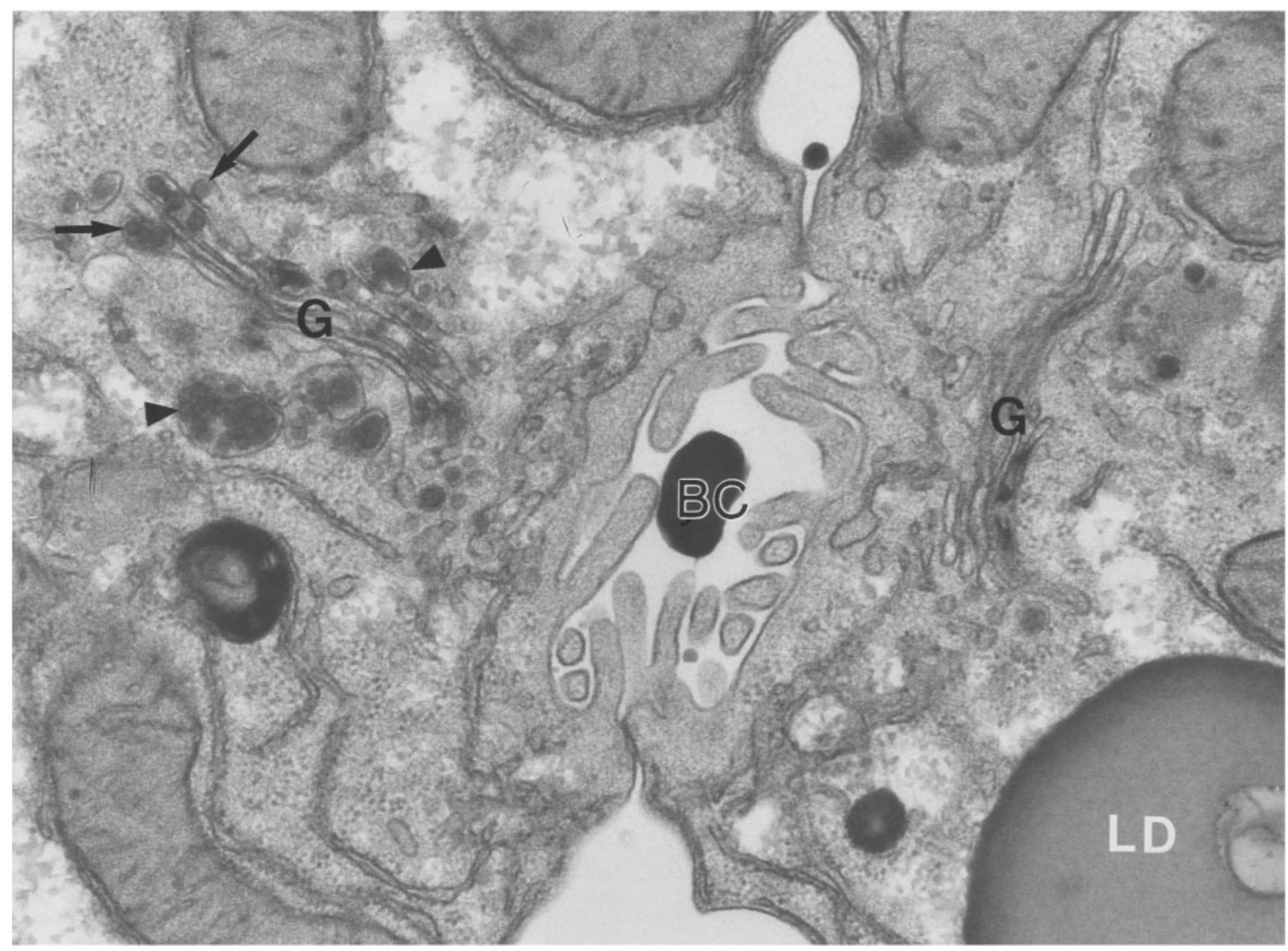

Figure 8

Electron micrograph of 2 adjoining hepatocytes from an $M t t p^{\Delta / \Delta}$ mouse (a plpC-treated Mttplox/flox/Mx1-Cre mouse), providing an example of the rare ultrastructural heterogeneity in hepatocytes from these mice. The cell to the right of the bile canaliculus (BC), almost certainly an $M t t p^{\Delta / \Delta}$ hepatocyte, contained numerous cytosolic lipid droplets (LD) and a complete absence of VLDL-sized, lipid-staining particles within the Golgi apparatus (G). The cell on the right of the BC was typical of $>98 \%$ of hepatocytes in $M t t p^{\Delta \Delta}$ mice. The cell to the left of the BC, likely a Mttp ${ }^{\Delta / \text { flox }}$ hepatocyte, had few or no cytosolic lipid droplets and small (300-550 A) VLDL in Golgi (G) stacks (arrows) and secretory vesicles (arrowheads). We occasionally observed some irregularly shaped lipid-staining "smudges" in the Golgi stacks of Mttp ${ }^{\Delta / \Delta}$ hepatocytes; the nature of this particulate matter is not known. Less than $2 \%$ of hepatocytes in $M t t p^{\Delta \Delta}$ mice were similar to the cell on the left. These rare lipoprotein-producing cells almost certainly had not been converted $\mathrm{t} M t t p^{\Delta / \Delta}$ genotype. $\times 36,000$. 


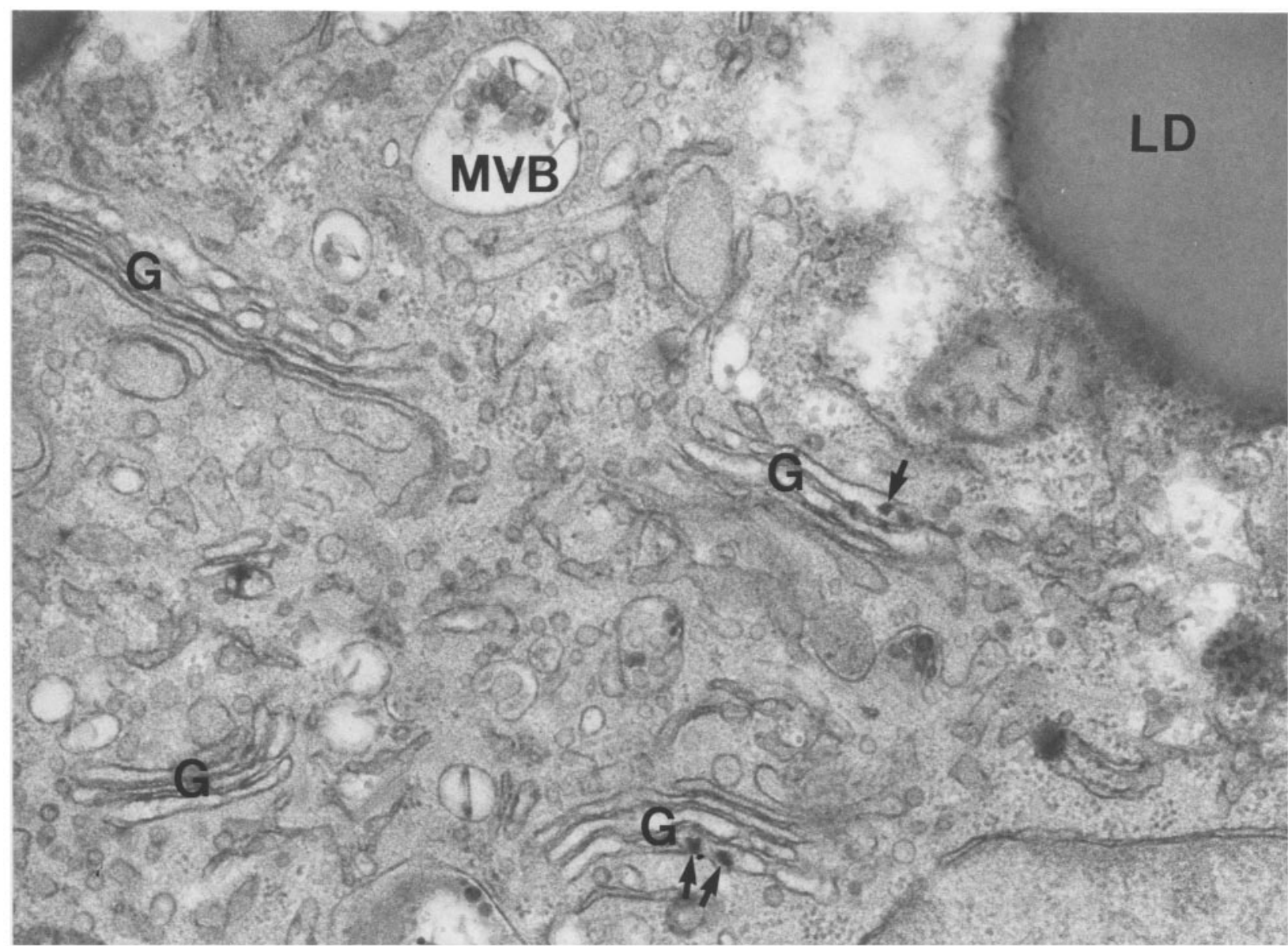

\section{Figure 9}

Electron micrograph of a hepatocyte from the liver of an Mttp ${ }^{\Delta / \Delta}$ mouse, illustrating the ultrastructural characteristics of the vast majority of hepatocytes. At the ultrastructural level, the most striking feature of the Mttp ${ }^{\Delta / \Delta}$ hepatocytes was the complete absence of VLDL-sized, lipid-staining particles within either the ER or the Golgi apparatus (G). Four Golgi complexes are imaged in this electron micrograph. The Mttp ${ }^{\Delta / \Delta}$ hepatocytes contained numerous cytosolic lipid droplets (LD). Small and irregularly shaped lipid-staining material was occasionally observed within the Golgi cisternae (arrows). The smooth ER is widely dispersed in hepatocytes. We scrutinized more than 100 electron micrographs of Mttp ${ }^{\Delta / \Delta}$ hepatocytes and did not observe round, VLDL-sized, lipid-staining particles in the ER. $\times 36,000$.

HDL-sized particles are far too small $(<150 \AA ̊)$ and cannot be clearly seen using electron microscopy. Of course, the hypothesis that $M t t p^{\Delta / \Delta}$ hepatocytes can secrete apo B48-containing HDL, but not apo B-48-containing VLDL, is supported strongly by the virtual absence of apo B48-containing VLDL in the plasma of $M t t p^{\Delta / \Delta}$ mice. Of note, primary mouse and rat hepatocytes normally secrete large amounts of apo B-48 in HDL-sized lipoproteins, in addition to apo B-48-containing VLDL (Veniant, M., et al., unpublished observations). Our studies suggest that these apo B-48-containing HDL-sized particles can be secreted fairly efficiently in the absence of MTP. If this proves to be the case, the effects of MTP deficiency on lipoprotein secretion would be similar to the effects of orotic acid (a pyrimidine biosynthetic intermediate). Orotic acid blocks the secretion of apo B-48-containing VLDL by interfering with the transport of lipoproteins from the ER to the Golgi apparatus, but has little effect on the secretion of apo B-48-containing HDL (50).

To our knowledge, possibility that small and dense apo B-48 particles might be secreted from hepatocytes in the absence of MTP has never been considered previ- ously. However, several prior observations lend credence to this scenario. First, Herscovitz et al. (51) reported apo B-41 secretion from C127 cells (a breast cancer cell line), even though those cells do not express MTP. Second, apo B-48 secretion appears to be more resistant than apo B-100 secretion to inhibition with MTP inhibitor drugs $(27,52,53)$. On the other hand, the suggestion that apo B-48 is secreted in the absence of MTP would be in conflict with earlier cell-culture studies that demonstrated that MTP expression was required for apo B secretion from cells that had been transfected with apo B-53 or apo B-41 expression vectors (54-56). The latter experiments, however, were performed with HeLa and Cos-7 cells, which do not normally synthesize or secrete lipoproteins. That consideration may be relevant, since it is conceivable that hepatocytes express another factor (either a lipid or another protein) that might assist in the secretion of small and dense apo B48 particles, even in the setting of MTP deficiency.

The liver-specific Mttp knockout mice have provided other lessons for understanding lipoprotein metabolism and for understanding abetalipoproteinemia. In our liver- 
specific knockout mice, VLDL and HDL levels were reduced substantially. We suspect that these 2 findings are mechanistically linked, since the lipolysis of triglyceride-rich lipoproteins provides substrates for the generation of HDL (57). In a prior study, we produced mice that lacked chylomicrons and documented a nearly identical 50\% reduction in HDL cholesterol levels (41). Considered together, these studies provide a compelling argument that the liver and the intestine contribute nearly equally to the maintenance of normal plasma HDL levels. From the perspective of understanding abetalipoproteinemia, we found that the absence of MTP did not result in a compensatory upregulation of hepatic apo $B$ mRNA levels, as had been suggested by several studies in humans (44-46). The absence of a change in Apob mRNA levels is in keeping with the fact that apo B mRNA levels typically change very little in response to a variety of other metabolic interventions (58).

The liver-specific Mttp knockout mice had moderate hepatic steatosis on a low-fat chow diet. One might reasonably expect the hepatic steatosis in the liver-specific knockout mice to be quite severe - perhaps more severe than that observed in humans with abetalipoproteinemia - since $M t t p^{\Delta / \Delta}$ mouse livers were required to deal with an onslaught of intestinally derived remnant lipoproteins. The livers of humans with abetalipoproteinemia, in contrast, do not face the burden of metabolizing intestinal lipoproteins. We found it intriguing that serum transaminases were normal in liver-specific Mttp knockout mice produced with the Mx1-Cre transgene approach and that the livers of those mice were devoid of any inflammatory infiltrate. These observations will probably be viewed as a hopeful sign for those interested in bringing MTP inhibitors to the marketplace as cholesterol-lowering agents (2). However, we believe that caution is required in interpreting the absence of hepatic inflammation; we have not yet followed liver histology in the liver-specific knockout mice for a prolonged period, nor have we evaluated the possibility that the steatosis might render the liver more susceptible to inflammation in response to a second insult.

The floxed Mttp allele produced in this study should be useful for understanding other aspects of MTP biology and for studies of atherogenesis. For example, generating intestine-specific Mttp knockout mice would make it possible to define the effects of MTP deficiency in that tissue and could provide an approach for defining mechanisms for the portal absorption of long-chain fatty acids, which is thought to occur in the absence of chylomicron secretion (59). Producing heart-specific Mttp knockout mice would provide an approach to understanding the physiologic importance of lipoprotein secretion by the myocardium $(3,4)$. Tissue-specific knockout mice might also provide an approach for understanding the significance of Mttp gene expression in the ovary and testis (60). Finally, the floxed Mttp allele provides an approach for abruptly changing LDL cholesterol levels in atherosclerosis-susceptible mice, such as the "apo B-100 only" LDL receptor-deficient mice (61). Sudden changes in LDL levels might provide an attractive model for studying the regression of atherosclerotic lesions without resorting to dietary interventions.
While this paper was under review, another group reported the development of liver-specific MTP knockout mice (62), using a floxed "MTP" allele and Cre adenovirus. Surprisingly, they observed no effect of the liverspecific MTP knockout on the total plasma triglyceride levels. In our $\mathrm{pIpC}$-treated $M t t p^{\text {flox/flox }} / \mathrm{Mx} 1-C r e$ mice, we observed significantly reduced plasma triglycerides. Also, they found a striking reduction in plasma apo B-48 levels, while we observed, on average, a $20 \%$ reduction. In some animals, we have occasionally observed $50 \%$ reduction in plasma apo B-48 levels, and that has generally occurred in our Cre adenovirus-treated animals. The reason for this apparent phenotypic difference is not obvious. No light or electron microscopy studies were provided in their publication.

\section{Acknowledgments}

We are grateful to our good friend and valued colleague Laura Flynn for the initial mapping of the Mttp gene. We proudly dedicate this paper to her. We thank T. Yu for blastocyst injections; J. Herz and R. Hammer (University of Texas-Southwestern, Dallas, Texas, USA) for the Mx1-Cre transgenic mice; F. Graham for the Cre adenovirus; D. Newland for assistance with histology; and R. Agah, R. Linnemann, and D. Dichek for assistance in preparing Cre adenovirus. This work was supported by National Institutes of Health grants HL-47660 and HL-41633 (to S.G. Young); a grant from the Deutsche Forschungsgemeinschaft and a postdoctoral research fellowship from the American Heart Association, Western States Affiliate (to M. Raabe); a grant-in-aid from the American Heart Association, Western States Affiliate (to R.L. Hamilton and J.S. Wong); a grant from the University of California-San Francisco Academic Senate Committee on Research (to R.L. Hamilton); and a Young Investigator Award from the University of California Tobacco Related Disease Research Program (to M.M. Véniant).

1. Gordon, D.A. 1997. Recent advances in elucidating the role of the microsomal triglyceride transfer protein in apolipoprotein B lipoprotein assembly. Curr. Opin. Lipidol. 8:131-137.

2. Wetterau, J.R., et al. 1998. An MTP inhibitor that normalizes atherogenic lipoprotein levels in WHHL rabbits. Science. 282:751-754

3. Nielsen, L.B., et al. 1998. Genes for apolipoprotein B and microsomal triglyceride transfer protein are expressed in the heart. Evidence that the heart has the capacity to synthesize and secrete lipoproteins. Circulation. 98:13-16.

4. Borén, J., Véniant, M.M., and Young, S.G. 1998. Apo B100-containing lipoproteins are secreted by the heart. J. Clin. Invest. 101:1197-1202.

5. Raabe, M., et al. 1998. Knockout of the abetalipoproteinemia gene in mice: reduced lipoprotein secretion in heterozygotes and embryonic lethality in homozygotes. Proc. Natl. Acad. Sci. USA. 95:8686-8691.

6. Walzem, R.L., Hansen, R.J., Williams, D.L., and Hamilton, R.L. 1999. Estrogen induction of VLDLy assembly in egg-laying hens. J. Nutr. 129:467S-472S

7. Wetterau, J.R., and Zilversmit, D.B. 1985. Purification and characterization of microsomal triglyceride and cholesteryl ester transfer protein from bovine liver microsomes. Chem. Phys. Lipids. 38:205-222.

8. Atzel, A., and Wetterau, J.R. 1993. Mechanism of microsomal triglyceride transfer protein catalyzed lipid transport. Biochemistry. 32:10444-10450.

9. Wetterau, J.R., et al. 1991. Protein disulfide isomerase appears necessary to maintain the catalytically active structure of the microsomal triglyceride transfer protein. Biochemistry. 30:9728-9735.

10. Wetterau, J.R., et al. 1992. Absence of microsomal triglyceride transfer protein in individuals with abetalipoproteinemia. Science. 258:999-1001.

11. Kane, J.P., and Havel, R.J. 1989. Disorders of the biogenesis and secretion of lipoproteins containing the B apolipoproteins. In The metabolic basis of inherited disease. 6th edition. C.R. Scriver, A.L. Beaudet, W.S. Sly, and D. Valle, editors. McGraw-Hill. New York, NY. 1139-1164.

12. Sharp, D., et al. 1993. Cloning and gene defects in microsomal triglyceride transfer protein associated with abetalipoproteinaemia. Nature. 365:65-69.

13. Rehberg, E.F., et al. 1996. A novel abetalipoproteinemia genotype. Identification of a missense mutation in the $97-\mathrm{kDa}$ subunit of the microsomal triglyceride transfer protein that prevents complex formation with 
protein disulfide isomerase. J. Biol Chem. 271:29945-29952.

14. Narcisi, T.M.E., et al. 1995. Mutations of the microsomal triglyceridetransfer-protein gene in abetalipoproteinemia. Am. J. Hum. Genet. 57:1298-1310.

15. Gregg, R.E., and Wetterau, J.R. 1994. The molecular basis of abetalipoproteinemia. Curr. Opin. Lipidol. 5:81-86.

16. Alexander, C.A., Hamilton, R.L., and Havel, R.J. 1976. Subcellular localization of B apoprotein of plasma lipoproteins in rat liver. J. Cell Biol. 69:241-263.

17. Hamilton, R.L., Erickson, S.K., and Havel, R.J. 1995. Nascent VLDL assembly occurs in two steps in the endoplasmic reticulum (ER) of hepatocytes. In Atherosclerosis X. F.P. Woodford, J. Davignon, and A. Sniderman, editors. Elsevier Science. Amsterdam, the Netherlands. 414-418.

18. Spring, D.J., et al. 1992. Lipoprotein assembly. Apolipoprotein B size determines lipoprotein core circumference. J. Biol. Chem. 267:14839-14845.

19. Cartwright, I.J., and Higgins, J.A. 1995. Intracellular events in the assembly of very-low-density-lipoprotein lipids with apolipoprotein B in isolated rabbit hepatocytes. Biochem. J. 310:897-907.

20. Innerarity, T.L., Borén, J., Yamanaka, S., and Olofsson, S.-O. 1996. Biosynthesis of apolipoprotein B48-containing lipoproteins. Regulation by novel post-transcriptional mechanisms. J. Biol. Chem. 271:2353-2356.

21. Gordon, D.A., et al. 1996. Inhibition of the microsomal triglyceride transfer protein blocks the first step of apolipoprotein B lipoprotein assembly but not the addition of bulk core lipids in the second step. J. Biol. Chem. 271:33047-33053.

22. Cartwright, I.J., et al. 1997. Investigation of the role of lipids in the assembly of very low density lipoproteins in rabbit hepatocytes. J. Lipid Res. 38:531-545.

23. Hamilton, R.L., et al. 1998. Chylomicron-sized lipid particles are formed in the setting of apolipoprotein B deficiency. J. Lipid Res. 39:1543-1557.

24. Borén, J., Rustaeus, S., and Olofsson, S.-O. 1994. Studies on the assembly of apolipoprotein B-100- and B-48-containing very low density lipoproteins in McA-RH7777 cells. J. Biol. Chem. 269:25879-25888.

25. Du, E.Z., et al. 1996. Translocation of apolipoprotein B across the endoplasmic reticulum is blocked in abetalipoproteinemia. J. Lipid Res. 37:1309-1315.

26. Gordon, D.A., Wetterau, J.R., and Gregg, R.E. 1995. Microsomal triglyceride transfer protein: a protein complex required for the assembly of lipoprotein particles. Trends Cell Biol. 5:317-321.

27. Wang, Y., McLeod, R.S., and Yao, Z. 1997. Normal activity of microsoma triglyceride transfer protein is required for the oleate-induced secretion of very low density lipoproteins containing apolipoprotein B from McARH7777 cells. J. Biol. Chem. 272:12272-12278.

28. Kühn, R., Schwenk, F., Aguet, M., and Rajewsky, K. 1995. Inducible gene targeting in mice. Science. 269:1427-1429.

29. Rajewsky, K., et al. 1996. Conditional gene targeting. J. Clin. Invest. 98:600-603.

30. O'Gorman, S., Dagenais, N.A., Qian, M., and Marchuk, Y. 1997. Protamine-Cre recombinase transgenes efficiently recombine target sequences in the male germ line of mice, but not in embryonic stem cells. Proc. Natl. Acad. Sci. USA. 94:14602-14607.

31. Rohlmann, A., Gotthardt, M., Hammer, R.E., and Herz, J. 1998 Inducible inactivation of hepatic LRP gene by Cre-mediated recombination confirms role of LRP in clearance of chylomicron remnants. $J$. Clin. Invest. 101:689-695.

32. Anton, M., and Graham, F.L. 1995. Site-specific recombination mediated by an adenovirus vector expressing the Cre recombinase protein: a molecular switch for control of gene expression. J. Virol. 69:4600-4606.

33.Rohlmann, A., et al. 1996. Sustained somatic gene inactivation by viral transfer of Cre recombinase. Nat. Biotechnol. 14:1562-1565.

34.Graham, F.L., and Prevec, L. 1991. Manipulation of adenovirus vectors. Methods Mol. Biol. 7:109-128.

35. McCormick, S.P.A., et al. 1996. Transgenic mice that overexpress mouse apolipoprotein B. Evidence that the DNA sequences controlling intestinal expression of the apolipoprotein B gene are distant from the structural gene. J. Biol. Chem. 271:11963-11970.

36. Linton, M.F., et al. 1993. Transgenic mice expressing high plasma concentrations of human apolipoprotein B100 and lipoprotein(a). J. Clin. Invest. 92:3029-3037.

37. Farese, R.V., Jr., et al. 1995. Knockout of the mouse apolipoprotein B gene results in embryonic lethality in homozygotes and protection against diet-induced hypercholesterolemia in heterozygotes. Proc. Natl. Acad. Sci. USA. 92:1774-1778.

38. Zlot, C.H., et al. 1999. Generation of monoclonal antibodies specific for mouse apolipoprotein B-100 in apolipoprotein B-48-only mice. J. Lipid Res. 40:76-84

39. Kim, E., et al. 1998. Dual mechanisms for the low plasma levels of trun- cated apolipoprotein B proteins in familial hypobetalipoproteinemia. Analysis of a new mouse model with a nonsense mutation in the Apob gene. J. Clin. Invest. 101:1468-1477.

40. Angermüller, S., and Fahimi, H.D. 1982. Imidazole-buffered osmium tetroxide: an excellent stain for visualization of lipids in transmission electron microscopy. Histochem. J. 14:823-835.

41. Young, S.G., et al. 1995. A genetic model for absent chylomicron formation: Mice producing apolipoprotein B in the liver, but not in the intestine. J. Clin. Invest. 96:2932-2946.

42. Wilcox, J.N., Gee, C.E., and Roberts, J.L. 1986. In situ cDNA:mRNA hybridization: development of a technique to measure mRNA levels in individual cells. Methods Enzymol. 124:510-533.

43. Sternberg, N., and Hamilton, D. 1981. Bacteriophage P1 site-specific recombination. I. Recombination between loxp sites. J. Mol. Biol. 150:467-486

44. Lackner, K.J., et al. 1986. Analysis of the apolipoprotein B gene and messenger ribonucleic acid in abetalipoproteinemia. J. Clin. Invest. 78:1707-1712.

45. Glickman, R.M., Glickman, J.N., Magun, A., and Brin, M. 1991. Apolipoprotein synthesis in normal and abetalipoproteinemic intestinal mucosa. Gastroenterology. 101:749-755.

46. Black, D.D., et al. 1991. Intestinal and hepatic apolipoprotein B gene expression in abetalipoproteinemia. Gastroenterology. 101:520-528.

47. Hermiston, M.L., Green, R.P., and Gordon, J.I. 1993. Chimeric-transgenic mice represent a powerful tool for studying how the proliferation and differentiation programs of intestinal epithelial cell lineages are regulated. Proc. Natl. Acad. Sci. USA. 90:8866-8870.

48. Gordon, J.I. 1993. Understanding gastrointestinal epithelial cell biology: Lessons from mice with help from worms and flies. Gastroenterology. 104:315-324.

49. Purcell-Huynh, D.A., et al. 1995. Transgenic mice expressing high levels of human apolipoprotein B develop severe atherosclerotic lesions in response to a high-fat diet. J. Clin. Invest. 95:2246-2257.

50. Hamilton, R.L., et al. 1986. Nascent high density lipoproteins from liver perfusates of orotic acid-fed rats. J. Lipid Res. 27:967-978.

51. Herscovitz, H., et al. 1995. Murine mammary-derived cells secrete the Nterminal $41 \%$ of human apolipoprotein B on high density lipoproteinsized lipoproteins containing a triacylglycerol-rich core. Proc. Natl. Acad. Sci. USA. 92:659-663.

52. van Greevenbroek, M.M.J., Robertus-Teunissen, M.G., Erkelens, D.W., and de Bruin, T.W.A. 1998. Participation of the microsomal triglyceride transfer protein in lipoprotein assembly in Caco-2 cells: interaction with saturated and unsaturated dietary fatty acids. J. Lipid Res. 39:173-185.

53. Haghpassand, M., Wilder, D., and Moberly, J.B. 1996. Inhibition of apolipoprotein B and triglyceride secretion in human hepatoma cells (HepG2). J. Lipid Res. 37:1468-1480.

54. Leiper, J.M., et al. 1994. Microsomal triglyceride transfer protein, the abetalipoproteinemia gene product, mediates the secretion of apolipoprotein B-containing lipoproteins from heterologous cells. J. Biol. Chem. 269:21951-21954.

55. Gordon, D.A., et al. 1994. Secretion of apolipoprotein B-containing lipoproteins from HeLa cells is dependent on expression of the microsomal triglyceride transfer protein and is regulated by lipid availability. Proc. Natl. Acad. Sci. USA. 91:7628-7632.

56. Wang, S., McLeod, R.S., Gordon, D.A., and Yao, Z. 1996. The microsomal triglyceride transfer protein facilitates assembly and secretion of apolipoprotein B-containing lipoproteins and decreases cotranslational degradation of apolipoprotein B in transfected COS-7 cells. J. Biol. Chem. 271:14124-14133.

57. Havel, R.J., and Kane, J.P. 1989. Structure and metabolism of plasma lipoproteins. In The metabolic basis of inherited disease. 6th edition. C.R. Scriver, A.L. Beaudet, W.S. Sly, and D. Valle, editors. McGraw-Hill. New York, NY. 1129-1138.

58. Young, S.G. 1990. Recent progress in understanding apolipoprotein B. Circulation. 82:1574-1594.

59. Dobbins, W.O., III. 1966. An ultrastructural study of the intestinal mucosa in congenital $\beta$-lipoprotein deficiency with particular emphasis upon the intestinal absorptive cell. Gastroenterology. 50:195-210.

60. Shoulders, C.C., et al. 1993. Abetalipoproteinemia is caused by defects of the gene encoding the $97 \mathrm{kDa}$ subunit of a microsomal triglyceride transfer protein. Hum. Mol. Genet. 2:2109-2116.

61. Véniant, M.M., et al. 1998 Assessing the roles of the two receptors for lipoprotein clearance in LDL receptor-deficient "apo B-48-only" and "apo B-100-only" mice. J. Clin. Invest. 102:1559-1568

62. Chang, B.H.-J., et al. 1999. Liver-specific inactivation of the abetalipoproteinemia gene completely abrogates very low density lipoprotein/low density lipoprotein production in a viable conditional knockout mouse. J. Biol. Chem. 274:6051-6055. 